\title{
The Minimal Length and the Quantum Partition Functions
}

\author{
M. Abbasiyan-Motlaq and Pouria Pedram* \\ Department of Physics, Science and Research Branch, \\ Islamic Azad University, Tehran, Iran
}

(Dated: June 29, 2021)

\begin{abstract}
We study the thermodynamics of various physical systems in the framework of the Generalized Uncertainty Principle that implies a minimal length uncertainty proportional to the Planck length. We present a general scheme to analytically calculate the quantum partition function of the physical systems to first order of the deformation parameter based on the behavior of the modified energy spectrum and compare our results with the classical approach. Also, we find the modified internal energy and heat capacity of the systems for the anti-Snyder framework.

PACS numbers: 04.60.Bc, 05.30.-d
\end{abstract}

Keywords: Generalized uncertainty principle, Minimal length, Thermostatistics

*Electronic address: p.pedram@srbiau.ac.ir 


\section{INTRODUCTION}

String theory, loop quantum gravity, quantum geometry, and black-hole physics as the candidates of quantum gravity all imply the existence of a minimal measurable length of the

order of the Planck length $\ell_{\mathrm{P}}=\sqrt{\frac{G \hbar}{c^{3}}} \approx 10^{-35} \mathrm{~m}$, where $G$ is Newton's gravitational constant [1 9]. To incorporate the idea of the minimal length with the laws of quantum mechanics, we need to modify the Heisenberg uncertainty principle to the Generalized (Gravitational) Uncertainty Principle (GUP). Based on the Heisenberg uncertainty principle, the minimal measurable length $\Delta X$ can be made arbitrarily small by letting $\Delta P$ grow correspondingly, but it fails near Planck energy scale where the corresponding Schwarzschild radius and Compton wavelength become approximately equal to the Planck length.

In the GUP framework, because of the modification of the commutation relations between the momentum and the position operators, all the Hamiltonians in quantum mechanics will be modified which results in the generalized Schrödinger equation in the quantum domain. Although the generalized Schrödinger equation is a high order differential equation and it is not easy to solve it, various problems such as the harmonic oscillator, the hydrogen atom, the Casimir effect, gravitational quantum well, and particles scattering have been investigated exactly or approximately in the GUP scenario [10 27].

Deliberation of many topics in theoretical physics are based on statistical approaches. In the GUP framework Fityo developed a classical method of the partition function evaluation based on modification of elementary cell of space volume according to modification of the commutation relation and investigated the thermodynamical properties of some physical systems [29]. The internal energy and the heat capacity of various physical systems are also calculated in Refs. [28-30]. Moreover, the effects of the minimal length on quantum partition function, internal energy and heat capacity using the exact treatment of the generalized Schrödinger equation is studied numerically in Ref. [18].

In this paper, we investigate the thermodynamical properties of the physical systems up to the first order of the GUP parameter. We present a general scheme to analytically calculate the modified partition function in term of ordinary partition function and the behavior of the modified energy spectrum of the physical systems. Here, we obtain the internal energy and heat capacity of one-dimensional ideal gas, harmonic oscillator, and Landau levels and compare our results with those obtained by the semiclassical approach. Also, we study these 
problems in anti-Snyder scenario based on our general scheme.

\section{THE GENERALIZED UNCERTAINTY PRINCIPLE}

In one dimension the modified commutation relation according to Kempf et al. is [11]

$$
[X, P]=i \hbar\left(1+\beta P^{2}\right)
$$

that leads to the following generalized uncertainty relation

$$
\Delta X \Delta P \geq \frac{\hbar}{2}\left(1+\beta(\Delta P)^{2}+\zeta\right)
$$

and a nonzero minimal measurable length $(\Delta X)_{\min }=\hbar \sqrt{\beta}=\sqrt{\beta_{0}} \ell_{\mathrm{P}}$. Here $\zeta$ is a constant and $\beta=\beta_{0} /\left(c^{2} M_{\mathrm{P}}^{2}\right)^{2}$ is the GUP parameter, $M_{\mathrm{P}}=\sqrt{\hbar c / G}$ is the Planck mass, and $\beta_{0}$ is a dimensionless parameter of the order of unity. Following Ref. [11] we can write $X$ and $P$ in the momentum space representation as

$$
\begin{aligned}
X \psi(p) & =i \hbar\left(1+\beta p^{2}\right) \frac{\partial \psi(p)}{\partial p} \\
P \psi(p) & =p \psi(p)
\end{aligned}
$$

where $X$ and $P$ are symmetric operators and exactly satisfy the commutation relation (11). However, in general, expressing the position operator as a composition of ordinary position and momentum operators would complicate the resulting differential equation. To overcome this problem and since the above representation is not unique, we can define the following quasi-position representation [18]

$$
\begin{aligned}
X \psi(\xi) & =\xi \psi(\xi), \\
P \psi(\xi) & =\frac{\tan \left(-i \hbar \sqrt{\beta} \partial_{\xi}\right)}{\sqrt{\beta}} \psi(\xi),
\end{aligned}
$$

To first order of GUP parameter, this representation can be written as [34]

$$
\begin{aligned}
& X \psi(\xi)=\xi \psi(\xi) \\
& P \psi(\xi)=-i \hbar\left(1-\frac{1}{3} \beta \hbar^{2} \frac{\partial^{2}}{\partial \xi^{2}}\right) \frac{\partial \psi(\xi)}{\partial \xi}
\end{aligned}
$$

Note that this representation is on the space of quasi-position wave functions not on the space of position wave functions. The quasi-position representation is firstly introduced by 
Kempf et al. in their seminal paper about twenty years ago [11] and extensively used by others during the past two decades. In the presence of a minimal uncertainty in position, the position operator is only symmetric and is no longer essentially self-adjoint. Nevertheless, the symmetricity implies that all expectation values are real. However, since the Heisenberg algebra representation contains no position eigenstates $|X\rangle$, there is no Hilbert space representation on position wave functions $\langle X \mid \psi\rangle$ in this algebra. Indeed, because the formal position eigenfunctions do not obey the minimal length uncertainty, they are not physical states.

Notice that, it is possible to project $|\psi\rangle$ on maximally localized states $\left|\psi_{\xi}^{m l}\right\rangle$ to find the probability amplitude for the maximally localized particle around the position $\xi$, i.e., $\xi=\left\langle\psi_{\xi}^{m l}|X| \psi_{\xi}^{m l}\right\rangle$. The maximal localization states are proper physical states and we can use them to define a 'quasi-position' representation. Although this representation does not diagonalize $X$, it has a direct interpretation in terms of position measurements. Therefore, the position and momentum operators $X, P$ can be written in terms of the multiplication and differentiation operators $\xi, p=-i \hbar \frac{d}{d \xi}$ that satisfy the ordinary commutation relations of quantum mechanics, namely, $[\xi, p]=i \hbar$. In Ref. [19], the maximally localized states are obtained in quasi-position representation given by Eqs. (5), 6 ).

In fact, we do not work with the same space of physical states with the same properties as we deal in ordinary quantum mechanics. For quasi-position wave functions and only for $\beta \rightarrow 0$, the scalar products (5, 66) agree with the ordinary scalar products on position space. Moreover, the quasi-position representation does not diagonalize the position operator in any domain of the symmetric operators $P^{2}$ and $X^{2}$. The direct physical interpretation of the quasi-position representation is the main preference of this representation. $\psi(\xi)$ denotes the probability amplitude for finding the particle which is maximally localized around the position $\xi$ and with the uncertainty $(\Delta X)_{\left|\psi_{\xi}^{m l}\right\rangle}=\hbar \sqrt{\beta}$ (see Refs. [18, 19] for more details).

To study the effects of GUP on the quantum mechanical systems, consider the Hamiltonian

$$
H=\frac{P^{2}}{2 m}+V(X)
$$

which using Eqs. (51) and (66) can be expressed as [18]

$$
H=\frac{\tan ^{2}(\sqrt{\beta} p)}{2 \beta m}+V(\xi)
$$


with the following perturbative form

$$
H=H_{0}+\sum_{n=3}^{\infty} \frac{(-1)^{n-1} 2^{2 n}\left(2^{2 n}-1\right)(2 n-1) B_{2 n}}{2 m(2 n) !} \beta^{n-2} p^{2(n-1)},
$$

where $H_{0}=\frac{P^{2}}{2 m}+V(\xi)$ and $B_{n}$ is the nth Bernouli number. Now in quasi-position space the Schrödinger equation generalizes to [18]

$$
-\frac{\hbar^{2}}{2 m} \frac{\partial^{2} \psi(\xi)}{\partial \xi^{2}}+\sum_{n=3}^{\infty} \alpha_{n} \hbar^{2(n-1)} \beta^{(n-2)} \frac{\partial^{2(n-1)} \psi(\xi)}{\partial \xi^{2(n-1)}}+V(\xi) \psi(\xi)=E \psi(\xi),
$$

where $\alpha_{n}=2^{2 n}\left(2^{2 n}-1\right)(2 n-1) B_{2 n} / 2 m(2 n)$ !. As we shall see, the presence of the additional terms in the modified Hamiltonian Eq. (11) leads to a positive shift in the energy spectrum of a particle. This modified differential equation is solved for the free particle, delta function potential, particle in a box and harmonic oscillator and their exact solutions are obtained [18].

\section{QUANTUM STATISTICAL MECHANICS}

All physical systems can be studied in quantum statistical mechanics by the partition function

$$
Z=\sum_{n} e^{-b E_{n}}
$$

where $E_{n}$ is energy spectrum, $b=\frac{1}{k_{B} T}, k_{B}$ is Boltzman's constant, and $T$ is the temperature. Since in the GUP scenario the generalized Schrödinger equation becomes a high order differential equation, it is not an easy task to solve it in general. However, for some specific problems this equation is solved and the solutions are already obtained [11, 13, 18, 32, 33]. For instance, for a particle with mass $m$ that is confined in an infinite one-dimensional box with length $L$, the corresponding energy eigenvalues are [18]

$$
E_{n}=\frac{\tan ^{2} \sqrt{2 m \beta E_{n}}}{2 m \beta}
$$

where $E_{n}^{0}=\frac{n^{2} \pi^{2} \hbar^{2}}{2 m L^{2}}$ are the eigenvalues of the ordinary Schrödinger equation. To first order we have

$$
E_{n}=E_{n}^{0}+\frac{4 m \beta}{3} E_{n}^{0^{2}}
$$


Also for the Harmonic oscillator potential $V(x)=\frac{1}{2} m \omega^{2} x^{2}$ we obtain [11]

$$
\begin{aligned}
E_{n} & =\hbar \omega\left(n+\frac{1}{2}\right)\left(\sqrt{1+\frac{\gamma^{2}}{4}}+\frac{\gamma}{2}\right)+\frac{1}{2} \hbar \omega \gamma n^{2}, \\
& =E_{n}^{0}+\frac{1}{2} m \beta E_{n}^{0^{2}}+\frac{1}{8} \beta m \hbar^{2} \omega^{2}+\mathcal{O}\left(\beta^{2}\right),
\end{aligned}
$$

where $\gamma=m \hbar \omega \beta$ and $E_{n}^{0}=\hbar \omega\left(n+\frac{1}{2}\right)$. As it is shown in Ref. [31], for the general polynomial potential in the form $V(x)=|a| x^{2(j+1)}+b x^{2(j+1)-1}+c x^{2(j+1)-2}+\ldots$ the approximate energy spectrum for $n \gg 1$ is given by

$$
E_{n} \simeq E_{n}^{0}+\mathcal{O}(1) m \beta E_{n}^{0^{2}}
$$

Now to find the thermodynamical variables we need to compute the partition function (13). Suppose the energy levels of systems have the form

$$
E_{n}=E_{n}^{0}+\varepsilon E_{n}^{0^{2}}+\Delta
$$

then

$$
Z(b ; \varepsilon)=\sum_{n} \exp \left[-b\left(E_{n}^{0}+\varepsilon E_{n}^{0^{2}}+\Delta\right)\right] .
$$

We now define $Z^{0}(b) \equiv \sum_{n} \exp \left(-b E_{n}^{0}\right)$, so we have

$$
Z(b ; \varepsilon)=e^{-b \Delta} \sum_{m=0}^{\infty} \frac{(-b \varepsilon)^{m}}{m !} \frac{\partial^{2 m}}{\partial b^{2 m}} Z^{0}(b) .
$$

For $b \varepsilon \ll 1$ or $T \gg \frac{\varepsilon}{k_{B}}$ and up to the first order of $\varepsilon$ the above equations takes the form

$$
Z(b ; \varepsilon)=e^{-b \Delta}\left(Z^{0}(b)-b \varepsilon \frac{\partial^{2}}{\partial b^{2}} Z^{0}(b)\right) .
$$

For a system that consists of $N$ particles we can write

$$
\ln Z_{N}(b ; \varepsilon)=\ln Z_{N}^{0}(b)+N \ln \left[1-b \varepsilon \frac{\frac{\partial^{2}}{\partial b^{2}} Z^{0}(b)}{Z^{0}(b)}\right]-b \Delta .
$$

Now, for $1-b \varepsilon \frac{\frac{\partial^{2}}{\partial b^{2}} Z^{0}(b)}{Z^{0}(b)}>0$ and using $\ln (1-x) \simeq-x$ for small $x(x \ll 1)$, we obtain

$$
\ln Z_{N}(b ; \varepsilon)=\ln Z_{N}^{0}(b)-\varepsilon N b \frac{\frac{\partial^{2}}{\partial b^{2}} Z^{0}(b)}{Z^{0}(b)}-b \Delta .
$$

So the partition function to $\mathcal{O}(\varepsilon)$ is

$$
Z_{N}(b ; \varepsilon)=Z_{N}^{0}(b) \exp \left[-\varepsilon N b \frac{\frac{\partial^{2}}{\partial b^{2}} Z^{0}(b)}{Z^{0}(b)}-b \Delta\right] .
$$


Also the modified internal energy $E=-\frac{\partial}{\partial b} \ln Z$ and the heat capacity $C=\frac{\partial E}{\partial T}$, Eq. (24) are given by

$$
\begin{aligned}
& E=E^{0}+\varepsilon \frac{\partial}{\partial b}\left[N b \frac{\frac{\partial^{2}}{\partial b^{2}} Z^{0}(b)}{Z^{0}(b)}\right]+\Delta+\mathcal{O}\left(\beta^{2}\right), \\
& C=C^{0}+\varepsilon \frac{\partial}{\partial T} \frac{\partial}{\partial b}\left[N b \frac{\frac{\partial^{2}}{\partial b^{2}} Z^{0}(b)}{Z^{0}(b)}\right]+\mathcal{O}\left(\beta^{2}\right),
\end{aligned}
$$

where $E^{0}$ and $C^{0}$ are respectively the internal energy and the heat capacity in ordinary thermodynamics framework. Eqs. (26) and (27) show that the shift in these variables can be just calculated in terms of ordinary quantum partition function $Z^{0}(b)$. Moreover, because of Eq. (18), this method can be applied to a wide range of problems.

It is worth to note that, we can also use these results for anti-Snyder algebra [20]

$$
[X, P]=i \hbar\left(1-\beta P^{2}\right)
$$

then by $\varepsilon \rightarrow-\varepsilon$, Eqs. (26) and (27) become

$$
\begin{aligned}
& E=E^{0}-\varepsilon \frac{\partial}{\partial b}\left[N b \frac{\frac{\partial^{2}}{\partial b^{2}} Z^{0}(b)}{Z^{0}(b)}\right]+\Delta+\mathcal{O}\left(\beta^{2}\right), \\
& C=C^{0}-\varepsilon \frac{\partial}{\partial T} \frac{\partial}{\partial b}\left[N b \frac{\frac{\partial^{2}}{\partial b^{2}} Z^{0}(b)}{Z^{0}(b)}\right]+\mathcal{O}\left(\beta^{2}\right),
\end{aligned}
$$

where $E$ and $C$ are the internal energy and heat capacity of the physical systems in antiSnyder scenario, respectively.

\section{APPLICATIONS}

In the following subsections we investigate the effects of GUP on the one-dimensional ideal gas, harmonic oscillator and the Landau levels.

\section{A. One-dimensional ideal gas}

Consider $N$ noninteracting particles with mass $m$ confined in one-dimensional box with length $L$. From Eqs. (15) and (19) we obtain $\varepsilon=\frac{4}{3} m \beta, \Delta=0$, and in ordinary statistical mechanics we have [35]

$$
Z^{0}(b)=\frac{L(2 \pi m)^{1 / 2}}{h b^{1 / 2}}
$$


Using Eqs. (26), (27), and (31) we obtain

$$
\begin{aligned}
& E=\frac{1}{2} N k_{B} T-\beta N m k_{B}^{2} T^{2}+\mathcal{O}\left(\beta^{2}\right), \\
& C=\frac{1}{2} N k_{B}-2 \beta N m k_{B}^{2} T+\mathcal{O}\left(\beta^{2}\right),
\end{aligned}
$$

which are valid in the small temperature limit. Conditions

$$
1-b \varepsilon \frac{\frac{\partial^{2}}{\partial b^{2}} Z^{0}(b)}{Z^{0}(b)}>0, \quad b \varepsilon \frac{\frac{\partial^{2}}{\partial b^{2}} Z^{0}(b)}{Z^{0}(b)} \ll 1,
$$

impose a restriction on temperature $T$. In the case of one-dimensional ideal gas this restriction is

$$
\frac{4 \beta m}{3 k_{B}} \ll T<\frac{1}{\beta m k_{B}},
$$

where for an electron with mass $m \sim 10^{-30} \mathrm{~kg}$, we have $10^{-9} \mathrm{~K} \ll T<10^{54} \mathrm{~K}$. Note that, as the above equation indicates, the temperature cannot be too small. From Eqs. (32) and (33) it can be seen that to $\mathcal{O}(\beta)$ the change in the internal energy and heat capacity of the system are quadratic and linear functions of the temperature, respectively, and at $T=\frac{1}{4 \beta m k_{B}}$ the heat capacity goes to zero. This result is also obtained in semiclassical approach of Ref. [29]. By taking $\beta \rightarrow-\beta$ in Eqs. (32) and (33), for anti-Snyder GUP and in the small $T$ limit we have

$$
\begin{aligned}
& E=\frac{1}{2} N k_{B} T+\beta N m k_{B}^{2} T^{2}+\mathcal{O}\left(\beta^{2}\right), \\
& C=\frac{1}{2} N k_{B}+2 \beta N m k_{B}^{2} T+\mathcal{O}\left(\beta^{2}\right),
\end{aligned}
$$

In contrast to Eqs. (32) and (33) the above equations indicate the increasing behavior of both internal energy and heat capacity in this framework.

\section{B. Harmonic oscillator}

In ordinary quantum statistical mechanics, a one-dimensional single harmonic oscillator's partition function is given by

$$
Z^{0}(b)=\left[2 \sinh \left(\frac{b \hbar \omega}{2}\right)\right]^{-1}
$$

According to Eq. (17), $\varepsilon$ and $\Delta$ can be written as

$$
\varepsilon=\frac{1}{2} m \beta, \quad \Delta=\frac{1}{8} \beta m \hbar^{2} \omega^{2},
$$


and consequently, Eqs. (26) and (27) to first order of the GUP parameter become

$$
\begin{gathered}
E=\frac{1}{2} \hbar \omega \operatorname{coth}\left(\frac{1}{2} b \hbar \omega\right)+\frac{1}{4} \beta m \hbar^{2} \omega^{2}\left[\operatorname{coth}^{2}\left(\frac{b \hbar \omega}{2}\right)-b \hbar \omega \operatorname{coth}\left(\frac{b \hbar \omega}{2}\right) \sinh ^{-2}\left(\frac{b \hbar \omega}{2}\right)\right]+\mathcal{O}\left(\beta^{2}\right), \\
C=\frac{k_{B}\left(\frac{1}{2} b \hbar \omega\right)^{2}}{\sinh ^{2}\left(\frac{1}{2} b \hbar \omega\right)}+\frac{1}{2} \beta m \hbar^{3} \omega^{3}\left[\frac{2(\sinh (b \hbar \omega)-b \hbar \omega)-(b \hbar \omega) \cosh (b \hbar \omega)}{k_{B} T^{2}(\cosh (b \hbar \omega)-1)^{2}}\right]+\mathcal{O}\left(\beta^{2}\right)
\end{gathered}
$$

The condition $1-b \varepsilon \frac{\frac{\partial^{2}}{\partial b^{2}} Z^{0}(b)}{Z^{0}(b)}>0$ in this case becomes

$$
b\left(\frac{1}{2} \beta m\right)\left(\frac{\hbar^{2} \omega^{2}}{4}\right)\left(-1+2 \operatorname{coth}^{2}\left(\frac{b \hbar \omega}{2}\right)\right)<1,
$$

which for $\hbar \omega \ll k_{B} T$ we obtain $\beta m k_{B} T<1$. Then the temperature condition (34) is given by

$$
\frac{\beta m}{2 k_{B}} \ll T<\frac{1}{\beta m k_{B}} .
$$

Therefore, in the small $T$ limit, $E$ and $C$ read

$$
\begin{aligned}
& E=k_{B} T-m \beta k_{B}^{2} T^{2}+\mathcal{O}\left(\beta^{2}\right), \\
& C=k_{B}-2 m \beta k_{B}^{2} T+\mathcal{O}\left(\beta^{2}\right) .
\end{aligned}
$$

Again, the heat capacity tends to zero at $T=\frac{1}{2 \beta m k_{B}}$ in agreement with Ref. [29]. Similarly, for anti-Snyder GUP we have

$$
\begin{aligned}
& E=\frac{1}{2} \hbar \omega \operatorname{coth}\left(\frac{1}{2} b \hbar \omega\right)+\frac{1}{4} \beta m \hbar^{2} \omega^{2}\left[\operatorname{coth}^{2}\left(\frac{b \hbar \omega}{2}\right)-b \hbar \omega \operatorname{coth}\left(\frac{b \hbar \omega}{2}\right) \sinh ^{-2}\left(\frac{b \hbar \omega}{2}\right)\right]+\mathcal{O}\left(\beta^{2}\right), \\
& C=\frac{k_{B}\left(\frac{1}{2} b \hbar \omega\right)^{2}}{\sinh ^{2}\left(\frac{1}{2} b \hbar \omega\right)}+\frac{1}{2} \beta m \hbar^{3} \omega^{3}\left[\frac{2(\sinh (b \hbar \omega)-b \hbar \omega)-(b \hbar \omega) \cosh (b \hbar \omega)}{k_{B} T^{2}(\cosh (b \hbar \omega)-1)^{2}}\right]+\mathcal{O}\left(\beta^{2}\right) .
\end{aligned}
$$

So in the small $T$ limit we find

$$
\begin{aligned}
& E=k_{B} T+m \beta k_{B}^{2} T^{2}+\mathcal{O}\left(\beta^{2}\right), \\
& C=k_{B}+2 m \beta k_{B}^{2} T+\mathcal{O}\left(\beta^{2}\right) .
\end{aligned}
$$

We observe that the heat capacity does not tend to zero for this case.

\section{The Landau levels}

Consider the problem of a particle of mass $m$ and charge $e$ in a constant magnetic field $\vec{B}=B \hat{k}$, with potential $\vec{A}=B x \hat{j}$ in the Landau gauge. The expression for the Hamiltonian 
is

$$
H^{0}=\frac{1}{2 m}(\vec{p}-e \vec{A})
$$

and the eigenfunctions and the eigenvalues of the Schrödinger equation are [10]

$$
\psi_{k, n}(x, y)=e^{i k y} \phi_{n}\left(x-x_{0}\right)
$$

and

$$
E_{n}^{0}=\hbar \omega_{c}\left(n+\frac{1}{2}\right), \quad n \in N
$$

where $\omega_{c}=\frac{e B}{m}$ is the cyclotron frequency and $\phi_{n}$ are the harmonic oscillator eigenfunctions. In GUP framework and to the first order of the GUP parameter, the Hamiltonian of this system is given by [10]

$$
H=H^{0}+4 \beta m H^{0^{2}}
$$

The eigenvalues are therefore

$$
E_{n}=E_{n}^{0}+4 \beta m E_{n}^{0^{2}}
$$

and $\frac{\Delta E_{n}}{E_{n}^{0}}=4 \beta m \hbar \omega_{c}\left(n+\frac{1}{2}\right)$. In ordinary quantum statistical mechanics, the partition function of this system is obtained as $Z^{0}(b)=\left[2 \sinh \left(\frac{b \hbar \omega_{c}}{2}\right)\right]^{-1}$. In analogous to harmonic oscillator, we obtain the following expression for the internal energy and the heat capacity of this system and to first order of the GUP parameter as

$$
\begin{aligned}
& E=E^{0}+\beta m \hbar^{2} \omega_{c}^{2}\left[-1+2 \operatorname{coth}^{2}\left(\frac{b \hbar \omega_{c}}{2}\right)-2 b \hbar \omega_{c} \operatorname{coth}\left(\frac{b \hbar \omega_{c}}{2}\right) \sinh ^{-2}\left(\frac{b \hbar \omega_{c}}{2}\right)\right]+\mathcal{O}\left(\beta^{2}\right),(55) \\
& C=C^{0}+4 \beta m \hbar^{3} \omega_{c}^{3}\left[\frac{2\left(\sinh \left(b \hbar \omega_{c}\right)-b \hbar \omega_{c}\right)-\left(b \hbar \omega_{c}\right) \cosh \left(b \hbar \omega_{c}\right)}{k_{B} T^{2}\left(\cosh \left(b \hbar \omega_{c}\right)-1\right)^{2}}\right]+\mathcal{O}\left(\beta^{2}\right)
\end{aligned}
$$

where $E^{0}=\frac{1}{2} \hbar \omega_{c} \operatorname{coth}\left(\frac{1}{2} b \hbar \omega_{c}\right)$ and $C^{0}=\frac{k_{B}\left(\frac{1}{2} b \hbar \omega_{c}\right)^{2}}{\sinh ^{2}\left(\frac{1}{2} b \hbar \omega_{c}\right)}$. Now, for $\hbar \omega_{c} \ll k_{B} T$ the temperature condition (34) reads

$$
\frac{4 \beta m}{k_{B}} \ll T<\frac{1}{\beta m k_{B}} .
$$

For this case, the above relations in the small $T$ limit simplify to

$$
\begin{aligned}
& E=k_{B} T-8 \beta m k_{B}^{2} T^{2}+\mathcal{O}\left(\beta^{2}\right), \\
& C=k_{B}-16 \beta m k_{B}^{2} T+\mathcal{O}\left(\beta^{2}\right) .
\end{aligned}
$$

Note that although the energy spectrum depends on the magnetic field $B$, the above equation shows that for $\hbar \omega_{c} \ll k_{B} T$ the internal energy is independent of the magnetic field. Again, 
for the anti-Snyder GUP we have

$$
\begin{aligned}
& E=E^{0}-\beta m \hbar^{2} \omega_{c}^{2}\left[-1+2 \operatorname{coth}^{2}\left(\frac{b \hbar \omega_{c}}{2}\right)-2 b \hbar \omega_{c} \operatorname{coth}\left(\frac{b \hbar \omega_{c}}{2}\right) \sinh ^{-2}\left(\frac{b \hbar \omega_{c}}{2}\right)\right]+\mathcal{O}\left(\beta^{2}\right),(60) \\
& C=C^{0}-4 \beta m \hbar^{3} \omega_{c}^{3}\left[\frac{2\left(\sinh \left(b \hbar \omega_{c}\right)-b \hbar \omega_{c}\right)-\left(b \hbar \omega_{c}\right) \cosh \left(b \hbar \omega_{c}\right)}{k_{B} T^{2}\left(\cosh \left(b \hbar \omega_{c}\right)-1\right)^{2}}\right]+\mathcal{O}\left(\beta^{2}\right)
\end{aligned}
$$

and

$$
\begin{aligned}
& E=k_{B} T+8 \beta m k_{B}^{2} T^{2}+\mathcal{O}\left(\beta^{2}\right), \\
& C=k_{B}+16 \beta m k_{B}^{2} T+\mathcal{O}\left(\beta^{2}\right),
\end{aligned}
$$

in the small $T$ limit.

\section{CONCLUSIONS}

In this paper, we have studied a GUP framework that implies a nonzero minimal length uncertainty and presented an analytic method to find the effects of GUP on the thermodynamical properties of the physical systems. We applied the method on one-dimensional ideal gas, harmonic oscillator, and Landau levels and obtained the modified partition functions, internal energies, and the heat capacities. We showed that up to the first order of the deformation parameter, the shift in the internal energy and heat capacity are quadratic and linear functions of the temperature, respectively. In particular, the minimal length causes the heat capacity tends to zero at some finite temperature in correspondence with the classical result. We also studied the thermodynamics of these systems in the anti-Snyder framework where the heat capacity represented a non-vanishing behavior.

[1] S. Hossenfelder, Living Rev. Relativity 16, 2 (2013).

[2] E. Witten, Phys. Today 49, 24 (1996).

[3] D. Amati, M. Ciafaloni and G. Veneziano, Phys. Lett. B 216, 41 (1989).

[4] C.A. Mead, Phys. Rev. B 849, 135 (1964).

[5] D.J. Gross, P.F. Mende, Nucl. Phys. B 303, 407 (1988).

[6] L.J. Garay, Int.J. Mod. Phys. A 10, 145 (1995).

[7] M. Maggiore, Phys. Lett. B 304, 65 (1993). 
[8] M. Kato, Phys. Lett. B 245, 43 (1990).

[9] F. Scardigli, phys. Lett. B 452, 39 (1999).

[10] S. Das, E.C. Vagenas, Can. J. Phys. 87, 233 (2009).

[11] A. Kempf, G. Mangano and R.B. Mann, Phys. Rev. D 52, 1108 (1995).

[12] A. Kempf, J. Phys. A 30, 2093 (1997).

[13] L.N. Chang, D. Minic, N. Okamura and T. Takeuchi, Phys. Rev. D 65, 125027 (2002).

[14] C. Quesne, V.M. Tkachuk, J. Phys. A 36, 10373 (2003).

[15] C. Quesne, V.M. Tkachuk, J. Phys. A 37, 10095 (2004).

[16] C. Quesne, V.M. Tkachuk, J. Phys. A 38, 1747 (2005).

[17] F. Brau, J. Phys. A 32, 7691 (1999).

[18] P. Pedram, Phys. Rev. D 85, 024016 (2012).

[19] P. Pedram, Adv. High Energy Phys. 2013, 853696 (2013).

[20] S. Mignemi, Phys. Rev. D 84, 025021 (2011).

[21] M.M. Stetsko, V.M. Takachuk, Phys . Rev. A 74, 012101 (2006).

[22] M.M. Stetsko, Phys . Rev. A 74, 062105 (2006).

[23] F. Brau, F. Buisseret, Phys. Rev. D 74, 036002 (2006).

[24] Kh. Nouricer. J. Phys. A 38, 10027 (2005).

[25] U. Harbach, S. Hossenfelder, Phys. Lett. B 632, 379 (2006).

[26] S. Hossenfelder, Phys. Rev. D 73, 105013 (2006).

[27] M.M. Stetsko, V.M. Takachuk, Phys. Rev. A 76, 012707 (2007).

[28] S.K. Rama, Phys, Lett. B 519, 903 (2001).

[29] T.V. Fityo, Phys. Lett. A 372, 5872 (2008).

[30] B. Vakili, M.A. Gorji, J. Stat. Mech. P10013 (2012).

[31] P. Pedram, Europhys. Lett. 89, 50008 (2010).

[32] K. Nozari, T. Azizi, Int. J. Quant. Inf. 3, 623 (2005).

[33] K. Nozari, P. Pedram, Eur. Phys. Lett. 92, 50013 (2010).

[34] S. Das, E.C. Vagenas. Phys. Rev. Lett. 101, 221301 (2008).

[35] R.K. Pathira, P.D. Beale, Statistical Mechanices (Academic, Boston, 2011). 